\title{
Simard, M. (2018). Le JAL : un demi-siècle de luttes et de débats. Bilan et perspectives nouvelles d'une expérience de développement communautaire en milieu rural. Rimouski, QC : Cahiers du GRIDEQ, 236 p.
}

André Joyal $^{\mathrm{a}}$

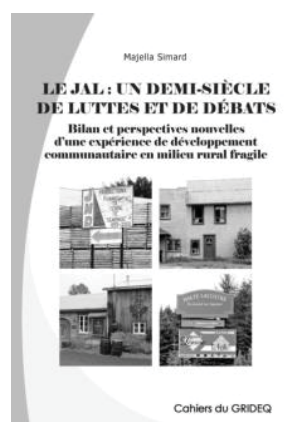

Majella Simard, spécialiste en développement régional, professeur au département d'histoire et de géographie de l'Université de Moncton, a opté pour un titre qui se veut aussi long que l'était celui d'un précédent volume. Le lecteur se trouve ainsi rapidement avisé du contenu, car c'est bien de ce que l'on qualifiait de développement communautaire dans les années 1970 dont il est ici question. Un développement initié par la population en vue de répondre à ses besoins. Les lecteurs de cette génération se rappelleront de l'acronyme JAL associé à cette expérience de développement inusitée mise en branle dans le Bas-Saint-Laurent sous le leadership de trois jeunes curés des paroisses Saint-Juste-du-lac, Auclair et Lejeune. L'acronyme s'explique par les premières lettres des trois villages. À une époque, on ne comptait plus le nombre de mémoires de maitrise rédigés sur le JAL par des étudiants de l'UQAR. Le JAL a fait couler beaucoup d'encre à la faveur de pas moins de 518 articles de journaux entre le jour de la Circoncision de 1971 et le jour de la Saint-Sylvestre de 2015. Et voilà, plus de 40 ans après les fameuses Opérations Dignité, l'auteur nous apprend qu'en fait, tout comme les trois mousquetaires d'Alexandre Dumas étaient quatre, les trois villages du JAL étaient quatre; en conséquence, on aurait dû parler du JALL, le dernier « L » pour Lots-Renversés (un tel nom ne s’invente pas...) situé en « banlieue » de Saint-Juste-du-Lac.

M. Simard parait en voie de devenir un spécialiste incontournable du Bas-Saint-Laurent en ce qui a trait au secteur associatif, à la participation citoyenne. L'auteur, né à Saint-André-de-Kamouraska, a fait ses trois cycles à l'UQAR sous le toit du pavillon principal de la rue Des Ursulines de Rimouski. Il en est sorti avec sous le bras une thèse de doctorat portant sur... le Bas-Saint-Laurent.

Cette fois, l'auteur s'est donné comme objectif d'évaluer la situation socioéconomique actuelle du JAL en prenant en considération, entre autres sources, les perceptions des acteurs locaux, jeunes et moins jeunes, sur l'évolution de leur milieu.

Afin que ses lecteurs, surtout les plus jeunes, comprennent bien l'intérêt que soulève le JAL, M. Simard, dans le chapitre 2 La problématique du JAL offre un rappel des faits en se rapportant aux suites des travaux du Bureau d'Aménagement de l'Est-du-Québec (BAEQ) réalisés dans la foulée de la loi fédérale de 1961 sur l'aménagement régional et le développement rural (ARDA) et dans le cadre d'une entente avec le gouvernement de Jean Lesage. De nombreuses propositions du BAEQ furent ignorées pour des raisons politiques reliées aux deux grands paliers de gouvernement. Par ailleurs, celle touchant la fermeture de plusieurs dizaines de petites municipalités marginales à travers la péninsule gaspésienne - souvent avec raison - n’est pas demeurée lettre morte. Le gouvernement de Robert Bourassa s'en rappellera lorsqu'au début des années 1970 on voudra accélérer la fermeture de certaines localités du Bas-Saint-Laurent. Cette fois, la résistance, sous la gouverne de précieux leaders, entreprirent de rallier les troupes sur le parvis de leur église. Ainsi, comme le signale le professeur de

a Professeur associé, Université du Québec à Trois-Rivières et membre du Centre de recherche et de développement territorial (CRDT) 
Moncton, à l'automne 70, 19 prêtres de la région Matapédia/Matane, avec l'appui de l'évêché de Rimouski, publièrent un manifeste de solidarité à la levée de boucliers qui se faisait sentir avec une détermination jusqu'alors inégalée. L'un d'entre eux, Gilles Roy, sera appelé à exercer un rôle déterminant dans les initiatives qui vont suivre les Opérations Dignité I, II et III.

Avec le chapitre 3 intitulé Cadrage théorique, M. Simard s'est attardé aux nombreux écrits de la part d'une gamme très variée d'auteurs qui, ces dernières décennies, se sont penchés sur la problématique du développement régional. Sans devoir pénétrer dans des approfondissements d'ordre théorique, le lecteur trouvera ici desinformations utiles sur la démographie, l'économie, les infrastructures, les politiques gouvernementales, et sur le fameux Capacity Building si cher à nos collègues du ROC ${ }^{1}$, que l'on est parvenu à traduire par une expression consacrée à la fin du siècle, "renforcement des capacités ». Cette dernière reviendra abondamment lorsque l'auteur dressera le bilan de la situation. S'en suivent d'autres concepts mis à la mode au tournant du siècle, contribuant ainsi, dans une certaine mesure, à justifier le titre du chapitre: gouvernance, innovation sociale (3.2.10), capital social (3.2.11), entrepreneuriat (3.2.12.1), capital socioterritorial (3.2.12.2) pour finir avec le développement territorial intégré.

Avec le chapitre 4, Analyse des résultats, le lecteur pourrait se demander de quels résultats il s'agit. De la recherche effectuée, faut-il le préciser? Tel un bénédictin dans son abbaye, plume d'oie à la main, l'auteur présente dans un premier temps de nombreuses données chiffrées telles celles issues du dépouillement des journaux. Le tableau 6 (p. 93) présente le nombre de recensions se rapportant à une trentaine d'initiatives réalisées sur le territoire. Suivent d'autres tableaux couvrant la période 1971-80. Ensuite, sans assommer le lecteur de données chiffrées, l'auteur couvre les périodes 1981-90, 1991-00, 2001-10 pour terminer avec les années 2011-15. On a beaucoup écrit sur le JAL et sur ce qu’il en reste.

Le plus intéressant nous est offert à la mi-parcours du livre avec la section 4.3 L'analyse perceptuelle se rapportant aux Acteurs locaux et régionaux est le cœur du sujet : L'expérience du JAL et ses retombées. Le tout débute ainsi : «Lorsque nous avons demandé à nos participants de raconter l'expérience qu'ils avaient du JAL et de relater comment ils l'avaient vécue, il était étonnant de constater que la majorité d'entre eux avaient toujours fraîchement en mémoire les différents événements relatifs à cette expérience » (p. 114). Même en 2015, pour plusieurs 1970 était hier. Diverses initiatives passées ou toujours en cours et de nouvelles font l'objet de fréquentes allusions, en particulier à L'Érablière la Grande Coulée.

Tous les répondants en conviennent, l'un des principaux facteurs de succès du mouvement jallois réside dans l'importance du leadership exercé par diverses catégories d'acteurs, dont, tel que mentionné plus haut, le clergé local. L'abbé Gilles Roy a joué un rôle de premier plan : «Gilles Roy a été une source d'inspiration. Il était un peu comme un mentor, un support moral avec ses idées et sa vision du développement » (entrevue no 6). Comme après la Conquête... «Le clergé monopolisait et s'occupait du territoire » (entrevue no 10)2. On aimera plus particulièrement la section La concertation: Principales difficultés rencontrées, où on lit : «Les gens du JAL se sont désociabilisés. Ils ne sont plus communautaires » (Entrevue no 1, p. 125). Là, comme ailleurs, la méfiance envers les néo-ruraux, même après plus de vingt ans, se fait sentir. « On sait ben, toi, tu n'es pas d'ici» combien de fois ils la reçoivent cette remarque 3 .

Parmi les nouvelles initiatives que le lecteur va suivre jusqu'à la fin de l'ouvrage, le projet de l'École de Cirque Funambule à Auclair occupe une place importante. Il en va de même pour la piste de BMX également mise de l'avant pour retenir les jeunes. On peut en dire autant en ce qui regarde le Théâtre amateur des Eaux-Claires (tant qu'à être à... Auclair). «Sans ces initiatives, je ne peux imaginer ce que serait le JAL aujourd'hui », entrevue no 8, (p. 166). 
Le Pacte rural, - qui, heureusement, n'a pas été complètement rayé d'un trait de crayon comme le fut la Politique nationale de la ruralité par le gouvernement Couillard à peine arrivé au pouvoir - fait l'objet d'intéressants commentaires. Mais, pour l'auteur, c'est trop peu. Il ne faut donc pas se surprendre de le voir prendre position à l'encontre d'un modèle marqué du sceau d'un néolibéralisme qui favorise la croissance à tout crin au détriment du développement (p. 186).

Ce livre, on l'aura compris, se destine aux gens des régions. Il intéressera les différents acteurs locaux qui, en région, feront les liens pertinents avec leur propre expérience.

\section{NOTES}

1 Rest of Canada : le Canada moins le Québec.

2 Signe des temps, lors de l'Université rurale québécoise, tenue en septembre 1999, à Trois-Pistoles, c'est un Gilles Roy laïcisé, marié et père de trois enfants qui dira que l'on ne peut plus songer à faire le développement comme dans les années 1970. Une nouvelle approche sera nécessaire. Il repose en paix depuis quelques années sans, comme on le voit, être tombé dans l'oubli.

3 Signalée à l'auteur de ces lignes à plusieurs reprises de la part de néo-ruraux où que ce soit : Canada, France, Brésil... 